\title{
The Fictitious Domain Method with Sharp Interface for Elasticity Systems with General Jump Embedded Boundary Conditions
}

\author{
Mohamed Kara ${ }^{1}$, Salim Mesbahi ${ }^{2, *}$ and Philippe Angot $^{3}$ \\ 1 Applied Mathematics Laboratory, Department of Mathematics, Faculty of Sciences, \\ Ferhat Abbas University, Setif, Algeria \\ 2 Fundamental and Numerical Mathematics Laboratory, Department of Mathematics, \\ Faculty of Sciences, Ferhat Abbas University, Setif, Algeria \\ ${ }^{3}$ Aix-Marseille Université, Institut de Mathématiques de Marseille, CNRS UMR 7373, \\ Marseille, France
}

Received 22 April 2019; Accepted (in revised version) 20 April 2020

\begin{abstract}
In framework of the fictitious domain methods with immersed interfaces for the elasticity problem, the present contribution is to study and numerically validate the jump-integrated boundary conditions method with sharp interface for the vector elasticity system discretized by a proposed finite volume method. The main idea of the fictitious domain approach consists in embedding the original domain of study into a geometrically larger and simpler one called the fictitious domain. Here, we present a cell-centered finite volume method to discretize the fictitious domain problem. The proposed method is numerically validated for different test cases. This work can be considered as a first step before more challenging problems such as fluid-structure interactions or moving interface problems.
\end{abstract}

AMS subject classifications: 65M55, 65N08, 65N85

Key words: Fictitious domain method, sharp interface, elasticity system, jump embedded boundary conditions, finite volume method.

\section{Introduction}

The conventional grid method approach to simulating transmission problems in heterogeneous elastic body with complex boundaries is to discretize the governing equations on a curvilinear mesh that conforms to the boundaries. The main advantages of this approach are that imposition of boundary conditions is greatly simplified and the scheme

${ }^{*}$ Corresponding author.

Emails: mohamed.kara@univ-setif.dz (M. Kara), salimbra@gmail.com (S. Mesbahi), philippe.angot@ univ-amu.fr (P. Angot) 
can be easily designed so as to maintain sufficient accuracy and conservation property. However, the geometrical complexity of immersed boundaries can adversely impact the stability, the convergence and the operation count of the solver. An alternative method approach which is gaining popularity in recent years is the fictitious domain methods approach where the governing equations are discretized on a uniform structure Cartesian mesh which does not conform to the immersed boundaries. This greatly simplifies mesh generation and retains the relative simplicity of the governing equations in Cartesian coordinates, (see $[1-8,10,11])$. In addition, since the Cartesian mesh scheme does not depend on the location of the immersed boundary, there is no need for remesching strategies and, thus, has a significant advantage in simulating problems with moving boundaries and complicated shapes. However, the difficulty in using the fictitious domain method in conjunction with a uniform structured Cartesian mesh scheme is in the position of the original boundary conditions at the immersed boundaries. Thus, to avoid this complication, an approximate interface $\Sigma_{h}$ of the original $\Sigma$ is constructed by a series of cell-sides that are cut by the boundary on which we apply an algebraic transmission boundary conditions proposed in our fictitious domain model-problem.

The numerical validation of a new fictitious domain method associated with general Jump Embedded Boundary Conditions (J.E.B.C) is proposed in the present paper. To solve the linear elasticity system governed by the given problem $(\widetilde{P})$ in the original domain $\widetilde{\Omega}$, a fictitious domain technique is used. The key issue here is to construct an extended imperfect transmission problem $(P)$ of $(\widetilde{P})$ defined in the extended domain $\Omega$ of the original physical domain $\widetilde{\Omega}$ in which its geometric shape is simpler than that of $\widetilde{\Omega}$ such that

$$
\Omega=\widetilde{\Omega} \cup \Sigma \cup \Omega_{e}
$$

where $\Omega_{e}$ is the external domain and $\Sigma$ the common interface between $\widetilde{\Omega}$ and $\Omega_{e}$, see Fig. 1. With an appropriate choice of the data and the transmission conditions in the auxiliary domain $\Omega_{e}$ and on the interface $\Sigma$ respectively, the transmission problem $(P)$ will be well-posed and the two problems are equivalents in the following sense : If $\mathbf{u}$ is a solution of the fictitious problem $(P)$ defined in the fictitious domain $\Omega$, then the restriction of the fictitious solution

$$
\tilde{\mathbf{u}}=\left.\mathbf{u}\right|_{\widetilde{\Omega}}
$$

or, at least, that $\widetilde{\mathbf{u}}_{\eta}=\left.\mathbf{u}\right|_{\widetilde{\Omega}}$ is a solution of the physical problem (original) $(\widetilde{P})$.

Our objective is to use a simple structured mesh in $\Omega$, e.g., a uniform Cartesian grid, independent of the shape of the immersed interface $\Sigma$, instead of using an adaptive mesh to which it becomes difficult to find fast and efficient solvers. The method is applied to several test cases for which an analytical and finite volume solution exists. Comparisons of the numerical and analytical results show a very good performance of the method. Our quest is to solve, with a fictitious domain method in $\Omega$, the following problem originally defined in $\widetilde{\Omega} \subset \Omega$ with a general boundary condition on $\Sigma$ : Find the displacement vector 
$\tilde{\mathbf{u}}$ and the symmetric stress tensor $\sigma=\left(\sigma_{i, j}\right)_{i, j=1}^{d}$ such that

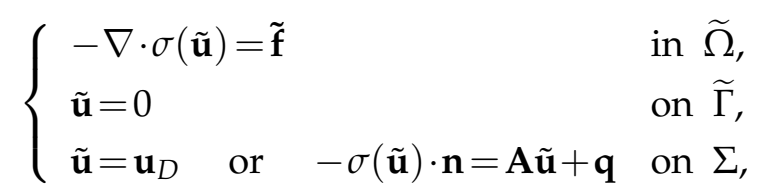

where $\tilde{\mathbf{f}} \in \mathbb{L}^{2}(\widetilde{\Omega})^{d}$ is the body force, $\mathbf{u}_{D} \in H^{1 / 2}(\Sigma)^{d}$ is the Dirichlet boundary condition, A $\in \mathbb{L}^{\infty}(\widetilde{\Sigma})^{d \times d}$ a uniformly positive matrix and $\mathbf{q} \in \mathbb{H}^{-1 / 2}(\Sigma)^{d}$ are given on $\Sigma$. Here $\lambda$ and $\mu \in \mathbb{L}^{\infty}(\widetilde{\Omega})$ are the Lamé coefficients, which we assume that

$$
\inf _{\widetilde{\Omega}} \phi(x)=\phi_{-}>0, \quad \phi_{+}=\sup _{\widetilde{\Omega}} \phi(x),
$$

where $\phi$ stands for $\lambda, \mu$ or $\rho$, and the constitutive equation in an isotropic linear elastic solid may be written as

$$
\sigma(\tilde{\mathbf{u}})=\lambda \nabla \cdot \tilde{\mathbf{u}} \mathbf{I}+2 \mu \varepsilon(\tilde{\mathbf{u}})
$$

here

$$
\varepsilon(\tilde{\mathbf{u}})=\frac{1}{2}\left[\nabla \tilde{\mathbf{u}}+(\nabla \tilde{\mathbf{u}})^{t}\right],
$$

is the linear strain tensor, $\mathbf{I}$ is the identity tensor, the Lamé coefficients $\lambda$ and $\mu$ are related to the its Young's modulus $E$ and the Poisson's ratio $v$ by the following relations:

$$
\lambda=\frac{E v}{(1-2 v)(1+v)}, \quad \mu=\frac{E}{2(1+v)} .
$$

In the next section the governing equations of the fictitious domain problem together with the treatment of the embedded original boundary conditions on the common interface $\Sigma$ are recalled. This is followed by a description of the finite volume discretization scheme and the solution algorithm. Finally, the method's capabilities are demonstrated by applying it to a number of test cases. Moreover, a particular scalar test problem with singular solution is also simulated.

\section{Fictitious model with jump embedded transmission conditions on $\Sigma$}

In this section, we present the governing equations of the fictitious model, together with the weak form and treatment of the embedded original boundary conditions.

Let the domain $\Omega \subset \mathbb{R}^{d}$ ( $d=2$ or 3 in practice) be an open bounded polygonal set. Let a regular enough interface $\Sigma \subset \mathbb{R}^{d-1}$ separate $\Omega$ into two disjointed sub domain $\widetilde{\Omega}$ and $\Omega_{e}$ such that $\Omega=\widetilde{\Omega} \cup \Sigma \cup \Omega_{e}$, the boundary of $\widetilde{\Omega}$ is defined by $\partial \widetilde{\Omega}=\widetilde{\Gamma} \cup \Sigma$ and the boundary of 


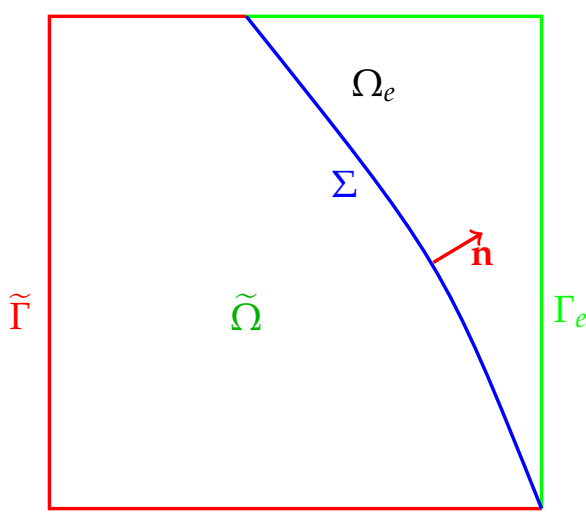

(a)

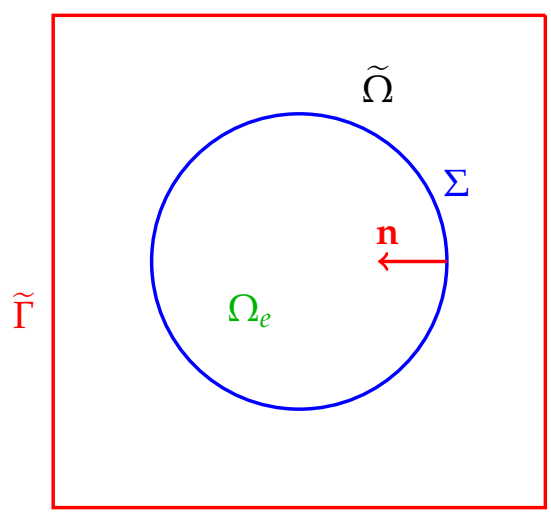

(b)

Figure 1: Two examples of original domain (physical) $\widetilde{\Omega}$ embedded in a fictitious domain $\Omega=\widetilde{\Omega} \cup \Omega_{e}$.

$\Omega$ is defined by $\partial \Omega=\widetilde{\Gamma} \cup \Gamma_{e}$. Let $\mathbf{n}$ be, either the outward unit normal vector on $\Gamma_{e}$, or the outward unit normal vector on $\Sigma$ oriented from $\widetilde{\Omega}$ to $\Omega_{e}$. For a function $\psi \in H^{1}\left(\widetilde{\Omega} \cup \Omega_{e}\right)$, let $\psi^{-}$and $\psi^{+}$be the traces of $\left.\psi\right|_{\Omega}$ and $\left.\psi\right|_{\Omega_{e}}$ on each side of $\Sigma$ respectively, $\left.\bar{\psi}\right|_{\Sigma}=\frac{1}{2}\left(\psi^{-}+\right.$ $\left.\psi^{+}\right)$the arithmetic mean of trace of $\psi$, and $\llbracket \psi \rrbracket_{\Sigma}=\left(\psi^{+}-\psi^{-}\right)$the jump of traces $\psi$ on $\Sigma$ oriented by $\mathbf{n}$.

\subsection{The strong form}

For the data $\mathbf{f} \in \mathbb{L}^{2}(\Omega)^{d}, \mathbf{g}$ and $\mathbf{h}$ given in $H^{-1 / 2}(\Sigma)^{d}$, we consider the elasticity system problem for the real-valued vector $\mathbf{u}=\left(u_{1}, \cdots, u_{d}\right)$ defined in $\Omega$ and includes immersed transmission conditions on $\Sigma$ links the trace of jumps of both the normal stress vector $\sigma(\mathbf{u}) \cdot \mathbf{n}=(\lambda(\nabla \cdot \mathbf{u}) \mathbf{I}+2 \mu \varepsilon(\mathbf{u})) \cdot \mathbf{n}$ and the traces of solution $\mathbf{u}$ through the interface $\Sigma:$

$$
\begin{cases}-\nabla \cdot \sigma(\mathbf{u})=\mathbf{f} & \text { in } \Omega=\widetilde{\Omega} \cup \Omega_{e}, \\ \mathbf{u}=0 & \text { on } \widetilde{\Gamma} \cup \Gamma_{e}, \\ \llbracket \sigma(\mathbf{u}) \cdot \mathbf{n} \rrbracket_{\Sigma}=\left.\mathbf{M} \overline{\mathbf{u}}\right|_{\Sigma}-\mathbf{h} & \text { on } \Sigma, \\ \left.\overline{\sigma(\mathbf{u}) \cdot \mathbf{n}}\right|_{\Sigma}=\mathbf{S} \llbracket \mathbf{u} \rrbracket_{\Sigma}-\mathbf{g} & \text { on } \Sigma,\end{cases}
$$

where the Lamé coefficients $\mu, \lambda$ and the transfer matrices $\mathbf{S}$ and $\mathbf{M}$ in the (J.E.B.C.) third and fourth equations of (2.1) on $\Sigma$ are measurable and bounded functions verifying ellipticity assumptions
$\mu, \lambda \in \mathbb{L}^{\infty}(\Omega), \exists \mu_{-}, \lambda_{-}>0, \mu>\mu_{-}, \lambda>\lambda_{-}$
a.e. in $\Omega$,
$\mathbf{M}, \mathbf{S} \in \mathbb{L}^{\infty}(\Sigma)^{d \times d}, \forall \xi \in \mathbb{R}^{d}, \xi^{t} \cdot \mathbf{M}(x) \cdot \xi>0, \xi^{t} \cdot \mathbf{S}(x) \cdot \xi>0$
a.e. on $\Sigma$. 
Moreover, in order to recover the original problem (1.1) in $\widetilde{\Omega}$, we choose $\mu$ and $\lambda$ a $\mathbb{L}^{\infty}$ extension of $\tilde{\mu}$ and $\tilde{\lambda}$ respectively in $\Omega$ and $\mathbf{f}$ a $\mathbb{L}^{2}$-extension of $\tilde{\mathbf{f}}$ in $\Omega$ such that

$$
\mu=\left\{\begin{array}{ll}
\tilde{\mu} & \text { in } \widetilde{\Omega}, \\
\mu_{e} & \text { in } \Omega_{e},
\end{array} \quad \lambda=\left\{\begin{array}{ll}
\tilde{\lambda} & \text { in } \widetilde{\Omega}, \\
\lambda_{e} & \text { in } \Omega_{e},
\end{array} \quad \mathbf{f}= \begin{cases}\tilde{\mathbf{f}} & \text { in } \widetilde{\Omega}, \\
\mathbf{f}_{e} & \text { in } \Omega_{e},\end{cases}\right.\right.
$$

as well as, the boundary condition the second equation in (2.1) on $\Gamma_{e}$ is chosen to ensure the solvability of the fictitious domain problem (2.1).

\subsection{The weak form}

We now introduce the Hilbert space :

$$
\mathbf{W}(\Omega)=\left\{\mathbf{v} \in \mathbb{L}^{2}(\Omega)^{d},\left.\mathbf{v}\right|_{\widetilde{\Omega}} \in H^{1}(\widetilde{\Omega})^{d} \text { and }\left.\mathbf{v}\right|_{\Omega_{e}} \in H^{1}\left(\Omega_{e}\right)^{d} ; \mathbf{v}=0 \text { on } \widetilde{\Gamma} \cup \Gamma_{e}\right\}
$$

equipped with the natural inner product and associated norm in $H^{1}(\Omega)^{d}$.

Let $\mathbf{u} \in \mathbf{W}$ satisfy the first equation in (2.1) and $\mathbf{f} \in L^{2}(\Omega)^{d}$, the weak form of the problem (2.1) can be written as : Find $\mathbf{u} \in \mathbf{W}$ such that

$$
a(\mathbf{u}, \mathbf{v})=l(\mathbf{v}), \quad \forall \mathbf{v} \in \mathbf{W},
$$

with

$$
\begin{aligned}
& a(\mathbf{u}, \mathbf{v})=\int_{\Omega} \sigma(\mathbf{u}): \nabla \mathbf{v} d x+\left.\left.\int_{\Sigma} \mathbf{M} \overline{\mathbf{u}}\right|_{\Sigma} \overline{\mathbf{v}}\right|_{\Sigma} d s+\int_{\Sigma} \mathbf{S} \llbracket \mathbf{u} \rrbracket_{\Sigma} \llbracket \mathbf{v} \rrbracket_{\Sigma} d s, \\
& l(\mathbf{v})=\int_{\Omega} \mathbf{f} \mathbf{v} d x+\left\langle\mathbf{h},\left.\overline{\mathbf{v}}\right|_{\Sigma}\right\rangle_{-\frac{1}{2}, \frac{1}{2}}+\left\langle\mathbf{g}, \llbracket \mathbf{v} \rrbracket_{\Sigma}\right\rangle_{-\frac{1}{2}, \frac{1}{2}} .
\end{aligned}
$$

Theorem 2.1 (Global solvability of the fictitious domain model with J.E.B.C.). If the ellipticity assumptions (A1), (A2) hold, the problem (2.1) with $\mathbf{f} \in \mathbb{L}^{2}(\Omega)^{d}$, and $\mathbf{g}$, $\mathbf{h} \in$ $L^{2}(\Sigma)^{d}$ has a unique weak solution $\mathbf{u} \in \mathbf{W}(\Omega)$ satisfying (2.2) for all $\mathbf{v} \in \mathbf{W}(\Omega)$, such that $\exists \alpha_{0}\left(\Omega, \Omega_{e}, \mu,\|\mathbf{S}\|,\|\mathbf{M}\|\right)>0$,

$$
\|\boldsymbol{u}\|_{\mathbb{W}} \leq \frac{1}{\alpha_{0}}\left(\|\mathbf{f}\|_{L^{2}(\Omega)}+c\left(\Omega, \Omega_{\mathcal{e}}\right)\left(\|\mathbf{g}\|_{-\frac{1}{2}, \Sigma}+\|\mathbf{h}\|_{-\frac{1}{2}, \Sigma}\right)\right) .
$$

Proof. We begin by deriving the weak form of the problem (2.1). With the first and second equations in (2.1) and using the Green-Stokes formula, $\langle\cdot, \cdot\rangle_{-\frac{1}{2}, \Sigma}$ being the duality pairing between $H^{-\frac{1}{2}}(\Sigma)^{d}$ and $H^{\frac{1}{2}}(\Sigma)^{d}$, we get respectively over $\widetilde{\Omega}$ and $\Omega_{e}$ :

$$
\begin{array}{lll}
\int_{\widetilde{\Omega}} \sigma(\mathbf{u}): \nabla \mathbf{v} d x-\left\langle\sigma(\mathbf{u})^{-} \cdot \mathbf{n}, \mathbf{v}^{-}\right\rangle_{-\frac{1}{2}, \Sigma}=\int_{\widetilde{\Omega}} \mathbf{f v} d x, & \forall \mathbf{v} \in \mathbf{W}, & \left.\mathbf{v}\right|_{\Omega_{e}}=0, \\
\int_{\Omega_{e}} \sigma(\mathbf{u}): \nabla \mathbf{v} d x+\left\langle\sigma(\mathbf{u})^{+} \cdot \mathbf{n}, \mathbf{v}^{+}\right\rangle_{-\frac{1}{2}, \Sigma}=\int_{\Omega_{e}} \mathbf{f} \mathbf{v} d x, & \forall \mathbf{v} \in \mathbf{W}, & \left.\mathbf{v}\right|_{\widetilde{\Omega}}=0 .
\end{array}
$$


Summing now the two previous equations yields :

$$
\int_{\Omega} \sigma(\mathbf{u}): \nabla \mathbf{v} d x+\left\langle\sigma(\mathbf{u})^{+} \cdot \mathbf{n}, \mathbf{v}^{+}\right\rangle_{-\frac{1}{2}, \Sigma}-\left\langle\sigma(\mathbf{u})^{-} \cdot \mathbf{n}, \mathbf{v}^{-}\right\rangle_{-\frac{1}{2}, \Sigma}=\int_{\Omega} \mathbf{f} \mathbf{v} d x, \quad \forall \mathbf{v} \in \mathbf{W} .
$$

Then, noticing that for any bilinear form $\langle\cdot, \cdot\rangle_{-\frac{1}{2}, \Sigma}$ defined on $\Sigma$, we have the equality below :

$$
\left\langle\mathbf{u}^{+}, \mathbf{v}^{+}\right\rangle_{\Sigma}-\left\langle\mathbf{u}^{-}, \mathbf{v}^{-}\right\rangle_{\Sigma}=\left\langle\llbracket \mathbf{u} \rrbracket_{\Sigma}, \overline{\mathbf{v}}_{\Sigma}\right\rangle_{\Sigma}+\left\langle\overline{\mathbf{u}}_{\Sigma}, \llbracket \mathbf{v} \rrbracket_{\Sigma}\right\rangle_{\Sigma}, \quad \forall \mathbf{u}, \mathbf{v},
$$

we obtain the following weak form in $\Omega$ :

$$
\int_{\Omega} \sigma(\mathbf{u}): \nabla \mathbf{v} d x+\left\langle\llbracket \sigma(\mathbf{u}) \cdot \mathbf{n} \rrbracket_{\Sigma}, \overline{\mathbf{v}}_{\Sigma}\right\rangle_{-\frac{1}{2}, \Sigma}+\left\langle\overline{\sigma(\mathbf{u}) \cdot \mathbf{n}_{\Sigma}}, \llbracket \mathbf{v} \rrbracket_{\Sigma}\right\rangle_{-\frac{1}{2}, \Sigma}=\int_{\Omega} \mathbf{f v} d x, \quad \forall \mathbf{v} \in \mathbf{W} .
$$

Then, using the jump transmission conditions i.e., the third and fourth equations in (2.1) on $\Sigma$, we get the nice weak formulation below: Find $\mathbf{u} \in \mathbf{W}$, sush that $\forall \mathbf{v} \in \mathbf{W}$,

$$
\int_{\Omega} \sigma(\mathbf{u}): \nabla \mathbf{v} d x+\int_{\Sigma} \mathbf{M} \bar{u}_{\Sigma} \bar{v}_{\Sigma}+\int_{\Sigma} \mathbf{S} \llbracket \mathbf{u} \rrbracket_{\Sigma} \llbracket \mathbf{v} \rrbracket_{\Sigma}=\int_{\Omega} \mathbf{f} \mathbf{v} d x+\left\langle\mathbf{h}, \overline{\mathbf{v}}_{\Sigma}\right\rangle_{-\frac{1}{2}, \Sigma}+\left\langle\mathbf{g}, \llbracket \mathbf{v} \rrbracket_{\Sigma}\right\rangle_{-\frac{1}{2}, \Sigma} .
$$

With the ellipticity assumptions $(A 1),(A 2)$, it is now easy to verify using the Korn inequality, in $\Omega, \Omega_{e}$ and standard trace lemmas that the left-hand side of the above nice weak formulation is a bilinear continuous and coercive form in $\mathbf{W} \times \mathbf{W}$, whereas the righthand is a linear continuous form in $\mathbf{W}$. Hence, by the Lax-Milgram theorem, we have existence and uniqueness of the weak solution $\mathbf{u}$ in $\mathbf{W}$.

Remark 2.1. Since $\nabla \cdot \sigma(\mathbf{u}) \in L^{2}(\Omega)^{d}$, then we can define $\sigma(\mathbf{u})_{\Sigma}^{ \pm}$in $H^{-1 / 2}(\Sigma)^{d}$.

\subsection{Treatment of the original boundary condition on $\Sigma$}

In this section, we will use for each kind of desired original boundary condition imposed on the immersed boundary $\Sigma$, only the particular variant with no exterior or surface control for both Fourier or Dirichlet boundary conditions respectively. These conditions will be enforced using a thin approximation of the immersed interface $\Sigma$, either the so-called Exterior interface $\Sigma^{E x t}$. or Cut interface $\Sigma^{C u t}$. by surface penalty of the Dirichlet boundary conditions and by choosing the transfer matrices in the first equation of system (2.3) which satisfies Eq. (2.5) or the third equation in (1.1) in the original problem, see [9,13-20].

\subsubsection{Embedded Fourier or Neumann boundary condition on $\Sigma$}

Let $-\sigma(\mathbf{u})_{\Sigma}^{-} \cdot \mathbf{n}$ and $-\sigma(\mathbf{u})_{\Sigma}^{+} \cdot \mathbf{n}$ be the traces of the normal stress on each side of $\Sigma$. In the previous fictitious domain model, we use the algebraic transmission conditions the third and fourth equations in (2.1) on $\Sigma$. the two quantities of the traces can be written in the following way:

$$
\left\{\begin{array}{l}
-\sigma(\mathbf{u})_{\Sigma}^{-} \cdot \mathbf{n}=\left(\mathbf{S}+\frac{1}{4} \mathbf{M}\right) \mathbf{u}_{\Sigma}^{-}-\left(\mathbf{S}-\frac{1}{4} \mathbf{M}\right) \mathbf{u}_{\Sigma}^{+}+\mathbf{g}-\frac{\mathbf{h}}{2} \quad \text { on } \Sigma \\
-\sigma(\mathbf{u})_{\Sigma}^{+} \cdot \mathbf{n}=-\left(\mathbf{S}+\frac{1}{4} \mathbf{M}\right) \mathbf{u}_{\Sigma}^{+}+\left(\mathbf{S}-\frac{1}{4} \mathbf{M}\right) \mathbf{u}_{\Sigma}^{-}+\mathbf{g}+\frac{\mathbf{h}}{2} \quad \text { on } \Sigma .
\end{array}\right.
$$


Table 1: Summary of the data in $\Omega_{e}$ and on $\Sigma$ for the J.E.B.C. Method.

\begin{tabular}{||ccc||}
\hline J.E.B.C. method & Parameters in $\Omega_{e}$ & Parameters on $\Sigma$ \\
\hline Fourier (F) & $\left.\lambda\right|_{\Omega_{e}}=\left.\mu\right|_{\Omega_{e}}=1,\left.\mathbf{f}\right|_{\Omega_{e}}=0$ & $\mathbf{M}=4 \mathbf{S}=2 \mathbf{A}, \mathbf{h}-\mathbf{g}=\mathbf{q}$ \\
Dirichlet (D1) & $\left.\lambda\right|_{\Omega_{e}}=\left.\mu\right|_{\Omega_{e}}=1,\left.\mathbf{f}\right|_{\Omega_{e}}=0$ & $\mathbf{M}=4 \mathbf{S}=\frac{2}{\eta}, \frac{\mathbf{h}}{2}-\mathbf{g}=\frac{1}{\eta} \mathbf{u}_{D}$ \\
Dirichlet (D2) & $\left.\lambda\right|_{\Omega_{e}}=\left.\mu\right|_{\Omega_{e}}=\frac{1}{\eta},\left.\mathbf{f}\right|_{\Omega_{e}}=\frac{1}{\eta} u_{e}$ & $\mathbf{S}=\frac{1}{\eta}, \mathbf{M}=\mathbf{g}=\mathbf{h}=\mathbf{0}$ \\
\hline
\end{tabular}

Then, with the particular choice $\mathbf{S}=\frac{1}{4} \mathbf{M}$ the system of Eq. (2.3) yields the Fourier boundary condition below, in this case the exterior fictitious domain problem and the interior (physical) one are independent :

$$
\begin{cases}-\sigma(\mathbf{u})_{\Sigma}^{-} \cdot \mathbf{n}=\frac{1}{2} \mathbf{M} \mathbf{u}_{\Sigma}^{-}+\mathbf{g}-\frac{\mathbf{h}}{2} & \text { on } \Sigma, \\ -\sigma(\mathbf{u})_{\Sigma}^{+} \cdot \mathbf{n}=-\frac{1}{2} \mathbf{M} \mathbf{u}_{\Sigma}^{+}+\mathbf{g}+\frac{\mathbf{h}}{2} & \text { on } \Sigma .\end{cases}
$$

When the Fourier or Neumann boundary condition in the third equation in (1.1) is desired for the original problem (1.1) in $\widetilde{\Omega}$, the following immersed boundary condition on $\Sigma$ must be satisfied by the solution of the fictitious domain problem in $\Omega$ :

$$
-\sigma(\mathbf{u})_{\Sigma}^{-} \cdot \mathbf{n}=\mathbf{A} \mathbf{u}_{\Sigma}^{-}+\mathbf{q} \text { on } \Sigma .
$$

This formulation enables us to deduce the transmission coefficients $\mathbf{S}, \mathbf{M}, \mathbf{g}$ and $\mathbf{h}$ on $\Sigma$ and the data $\mu, \lambda$ and $\mathbf{f}$ in $\Omega_{e}$ as well as on $\Sigma$, which are presented in the Table 1 .

Other variants are proposed there require either $\mathbf{u}_{\Sigma}^{+}$or $-\sigma(\mathbf{u})_{\Sigma}^{+} \cdot \mathbf{n}$ to be controlled by $\mathbf{L}^{2}$ or $\mathbf{H}^{1}$ volume penalty methods $[1-4,19]$ performed with the parameters in $\Omega_{e}$ for the convection-diffusion problem.

\subsubsection{Embedded Dirichlet boundary condition on $\Sigma$}

The Dirichlet boundary condition $\mathbf{u}_{\Sigma}^{-}=\mathbf{u}_{D}$ of the problem (1.1) is treated as a penalization of the previous Fourier boundary condition in the system of Eqs. (2.3) or (2.4). Indeed, the Fourier condition in the system of Eqs. (2.3) or (2.4) can be penalized by a surface penalty on $\Sigma$ with

$$
\mathbf{M}=\frac{2}{\eta} \mathbf{I} \longrightarrow+\infty \quad \text { and } \quad \frac{\mathbf{h}}{\mathbf{2}}-\mathbf{g}=\frac{1}{\eta} \mathbf{u}_{D},
$$

when $\eta \longrightarrow 0$, where $\eta$ is a real penalty parameter, (see (D1)) in the Table 1 . The second variant consists in using a $\mathbb{L}^{2}$ or $H^{1}$ volume penalty in the exterior domain $\Omega_{e}$ such that

$$
\lim _{\eta \longrightarrow 0} \mathbf{u}_{\eta \Sigma}^{+}=\mathbf{u}_{D}
$$

for the model with

$$
\mathbf{S}=\frac{1}{\eta} \mathbf{I} \longrightarrow+\infty, \quad \mathbf{M}=0, \quad \mathbf{h}=0
$$


and thus

as proposed in [7].

$$
\llbracket \mathbf{u} \rrbracket_{\Sigma} \longrightarrow 0, \quad \llbracket \boldsymbol{\phi} \cdot \mathbf{n} \rrbracket_{\Sigma}=0
$$

Remark 2.2. In the practice, the penalty parameter $\eta$ is chosen small enough, typically of the order of $10^{-7}$ such that the error due to the fictitious domain modeling in $\Omega$ for the original problem $\widetilde{\Omega}$ remains smaller than the error due to the numerical approximation.

\section{Characterization of the fictitious domain (original and auxiliary)}

For the numerical approximation of the fictitious domain problem (2.1), we use the finite volume methods because they are robust, relatively cheap, inherently conservative, and able to handle strong discontinuities in the differential operators. We consider the two-dimensional case $(d=2)$ in the Cartesian coordinates $(x, y)$, when the domain $\Omega$ is rectangular for a fictitious domain model. The discretization procedure is separated in two parts: discretization of the computational domain and equations discretization, see $[5-8,10-12,16,18,19]$.

In this part, we use the level set method which is a numerical technique for tracking moving interface, it is based upon the idea of representing the interface as a level set curve of higher-dimensional function $\phi(x, t)$. In keeping with the applications pursued in this paper, we only use the level set theory for representing the static interface $\Sigma$.

We decompose the computational domain (fictitious domain) $\Omega$ into two subdomains $\widetilde{\Omega}$ and $\Omega_{e}$ which are respectively associated to the part of the physical domain and auxiliary one. The principle is to define a distance function in the computational domain. If the interface of the physical domain is given by $\Sigma$, we can define by

$$
\varphi(x, 0)= \begin{cases}\left\|x-x_{\Sigma}\right\| & \text { in } \widetilde{\Omega}, \\ -\left\|x-x_{\Sigma}\right\| & \text { in } \Omega_{e},\end{cases}
$$

the characteristic function of the physical domain is easily obtained by looking at the sign of $\varphi\left(x_{i}, 0\right)$ in the nodes $x_{i}$ of the mesh :

$$
\chi_{p h}\left(x_{i}\right)= \begin{cases}1, & \text { if } \varphi\left(x_{i}, 0\right)>0, \\ 0, & \text { if } \varphi\left(x_{i}, 0\right)<0,\end{cases}
$$

finally, the characteristic function from the distance function in the control volume $K$ can be given by

$$
\left.\chi_{p h}\right|_{K}=\frac{\Sigma_{K}^{+}}{|\Sigma|_{K}},
$$

where $\Sigma_{K}^{+}$is the sum of the nodes of element $K$ which are $\varphi\left(x_{i}, 0\right)>0$, and $|\Sigma|_{K}$ is the sum of absolute values of $\varphi\left(x_{i}, 0\right)$. the value of function $\left.\chi_{s}\right|_{K}$ is 1 or 0 depending on whether all nodes of the element $K$ are positive or negative, but for the control volumes that are crossed by the embedding interface $\Sigma$, they, have a value between 0 and 1 . 


\section{Numerical results}

In order to illustrate the accuracy of the fictitious domain method for the elasticity problems, we have considered some problems with exact solutions. In that follows, we focus on 2-D problems. We present the results obtained from numerical simulations carried out on homogeneous and heterogeneous isotropic media. For the sake of simplicity, we carried out the calculations for the model where the Lamé Coefficients are constant, see $[1-4,9,16]$ and [17].

In the computation, the material properties are chosen as : Young's modulus $E=1.542$ and poisson's ratio $v=0.285$. These correspond to the Lamé's coefficients $\lambda=8000$ and $\mu=6000$. The errors between the numerical and analytic solutions to the following test problems can be appreciated by the calculation of either the $\mathbb{L}^{2}$-norm or the $\mathbb{L}^{\infty}$-norm in $\Omega_{h}$.

\subsection{Validation of the Finite Volume Scheme (F.V.S.) for the elasticity problem}

We begin by the validation of the finite volume scheme in the areas where the computational domain coincides with the physical one. For this purpose, the physical domain is the unit square $\Omega=[0,1] \times[0,1]$. The domain $\Omega$ is meshed by uniform square cells $K$ with a grid step varying from $h=\frac{1}{10}$ to $\frac{1}{120}$ and $\Sigma=\{(x, y) \in \Omega$ such that $x=1\}$.

The first test used to demonstrate the convergence of the proposed scheme, the nonhomogeneous Dirichlet and Fourier problems are considered and the Fig. 2 illustrates the relative $\mathbb{L}^{2}$-norm errors versus a discretisation step $h$ which shows the second order accuracy of this scheme.

\subsection{Validation of the fictitious domain method for two-dimensional linear elasticity system}

The conventional way of validating the efficient accuracy of the present scheme is to compute an elastic body which has both a L-shaped or a curved immersed boundary $\Sigma$ for which an analytical solution exists. In the numerical computations, the fictitious domain is the unit square, a convergence study is carried out using uniform rectangular meshes, the tests are performed for two different domains : L-shaped domain and a quarter disk domain. In the figures of errors, the plot of error in the energy norm versus the mesh spacing is shown on a log-log plot.

\subsubsection{First test problem: A L-shaped domain}

Dirichlet problem. First, we consider the following homogenous Dirichelet problem :

$$
\widetilde{\mathcal{P}}_{D H} \begin{cases}-\nabla \cdot \sigma(\tilde{u})=\tilde{f} & \text { in } \widetilde{\Omega}, \\ \tilde{u}=0 & \text { on } \widetilde{\Gamma} \\ \tilde{u}=0 & \text { on } \Sigma\left(u_{D}=0\right),\end{cases}
$$


Fourier (FP) and homogeneous Dirichlet problems (DP)

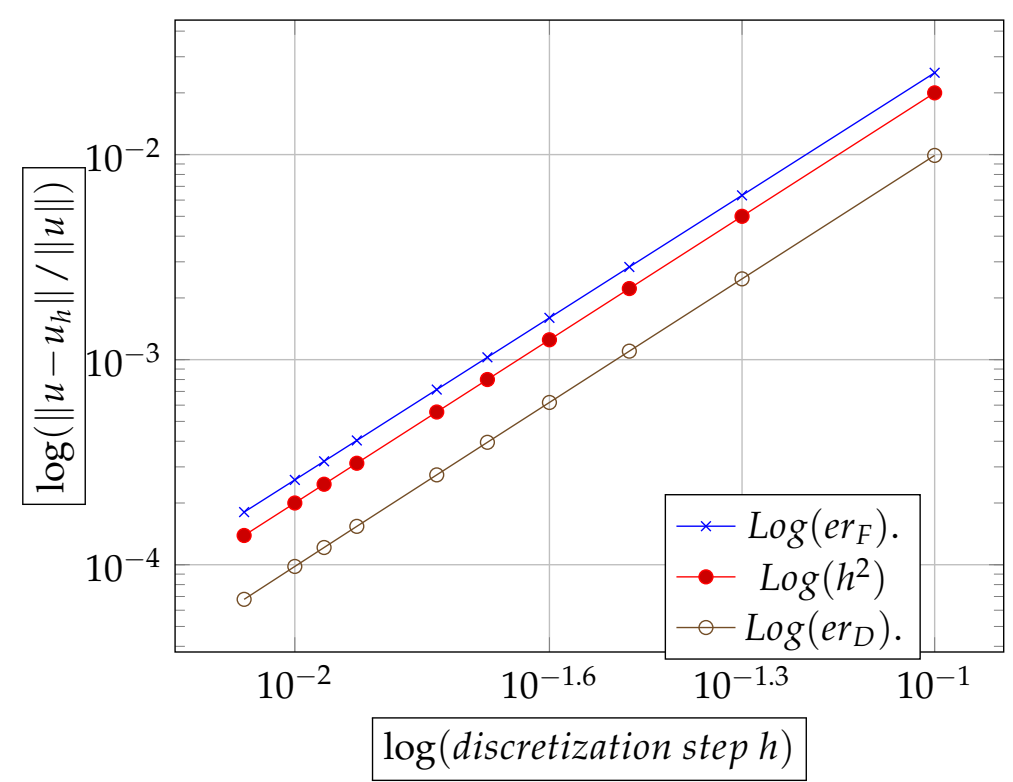

Figure 2: The second order accuracy of the schema for a (FP) and (DP) problems.

where $\Sigma=\Sigma_{x} \cup \Sigma_{y}$, such that

$$
\Sigma_{x}=\left\{\frac{(x, y)}{x}=\frac{1}{2} ; \frac{1}{2} \leq y \leq 1\right\} \quad \text { and } \quad \Sigma_{y}=\left\{\frac{(x, y)}{y}=\frac{1}{2} ; \frac{1}{2} \leq x \leq 1\right\},
$$

which has the analytic solution $\tilde{u}(x, y)=\tilde{v}(x, y)=\sin (2 \pi x) \sin (2 \pi y)$ in $\widetilde{\Omega}$, for the appropriate data for $f$.

Fig. 3 gives an example of the uniform grid and the immersed interface. This geometry is used for the test problems presented in this section.

The fictitious domain problem is solved in $\Omega$ with the J.E.B.C. method (D1), using the surface penalty on $\Sigma_{h}$ without exterior control in $\Omega_{e}$. In this case, the equation solved in $\Omega_{e, h}$ has no influence on the solution obtained in the physical domain. So, the equation parameters in $\Omega_{e, h}$ are arbitrary extensions of the original coefficients of physical problem. The Table 2 synthesizes the results obtained with the J.E.B.C. method (D1) and the parameter of penalization $\eta=o\left(10^{-7}\right)$. Note that with a Cartesian mesh on $\Omega$, the approximate interface $\Sigma_{h}$ of $\Sigma$ is exactly defined. So, the global error is defined as the sum of a modelling error (due to the penalization in the Dirichlet case) and numerical scheme error.

Fig. 4 shows the convergence of the numerical error with respect to the discretization step $h$ by J.E.B.C. method (D1) in the L-shaped domain $\widetilde{\Omega}$.

In order to validate the J.E.B.C. method (D1) for any Dirichlet boundary condition, 


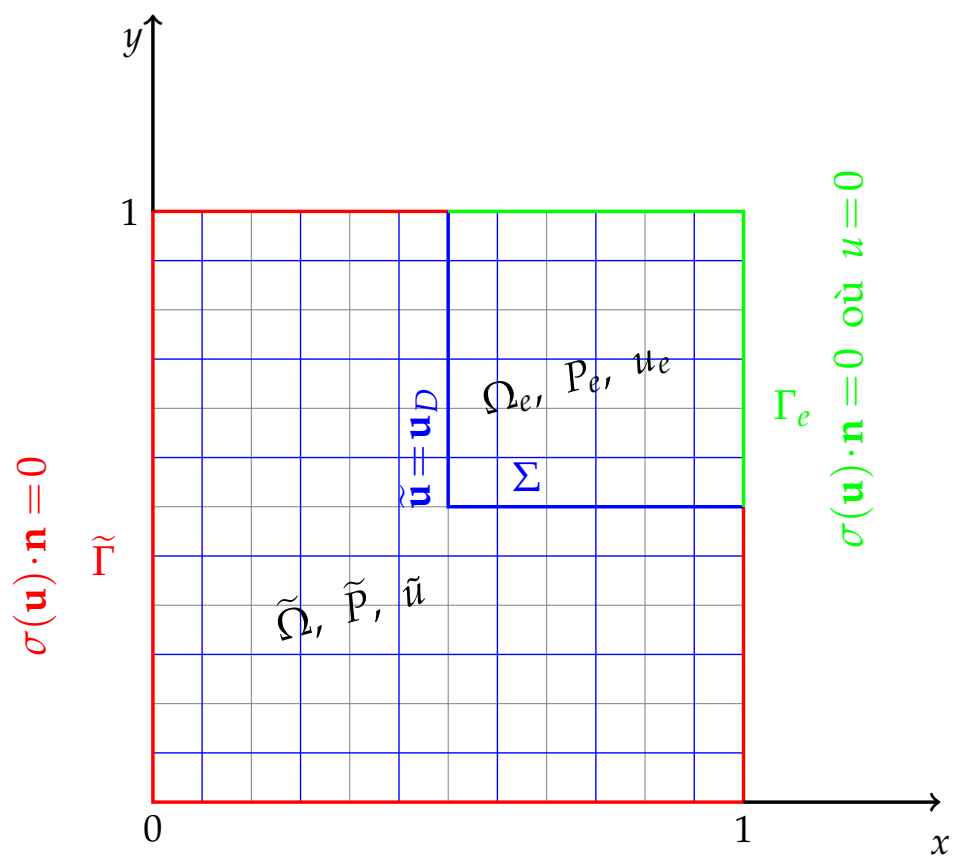

Figure 3: L-shaped physical domain immersed inside a fictitious domain $\Omega=\widetilde{\Omega} \cup \Sigma \cup \Omega_{\mathcal{e}}=[0,1] \times[0,1]$ in an uniform grid.

Table 2: Variation of relative $\mathbb{L}^{2}$ error norms according to the number of discretization points for Dirichlet condition in L-shaped domain.

\begin{tabular}{||c|c|c|c|c|c||}
\hline Mesh step $h$ & $1 / 10$ & $1 / 20$ & $1 / 40$ & $1 / 80$ & $1 / 140$ \\
\hline$\frac{\left\|u_{e x}-u_{h}\right\|}{\left\|u_{e x}\right\|}$ & $7.267270 e-2$ & $3.516438 e-2$ & $1.696973 e-2$ & $8.292075 e-3$ & $4.728605 e-3$ \\
\hline
\end{tabular}

we now study a non-homogeneous Dirichlet problem

$$
\left(\widetilde{\mathcal{P}}_{D N H}\right) \begin{cases}-\nabla \cdot \sigma(\tilde{u})=\tilde{f} & \text { in } \widetilde{\Omega}, \\ \tilde{u}=0 & \text { on } \widetilde{\Gamma}, \\ \tilde{u}=u_{D} & \text { on } \Sigma,\end{cases}
$$

where

$$
\begin{aligned}
& u_{D}=\left\{\begin{array}{l}
\frac{3}{16} \sin (\pi y), \\
\frac{3}{16} \sin (\pi y),
\end{array}\right. \\
& u_{D}=\left\{\begin{array}{l}
x(x-1) \sin (3 \pi / 4), \\
x(x-1) \sin (3 \pi / 4),
\end{array} \quad 3 / 4 \leq x \leq 1, \quad y=3 / 4,\right.
\end{aligned}
$$




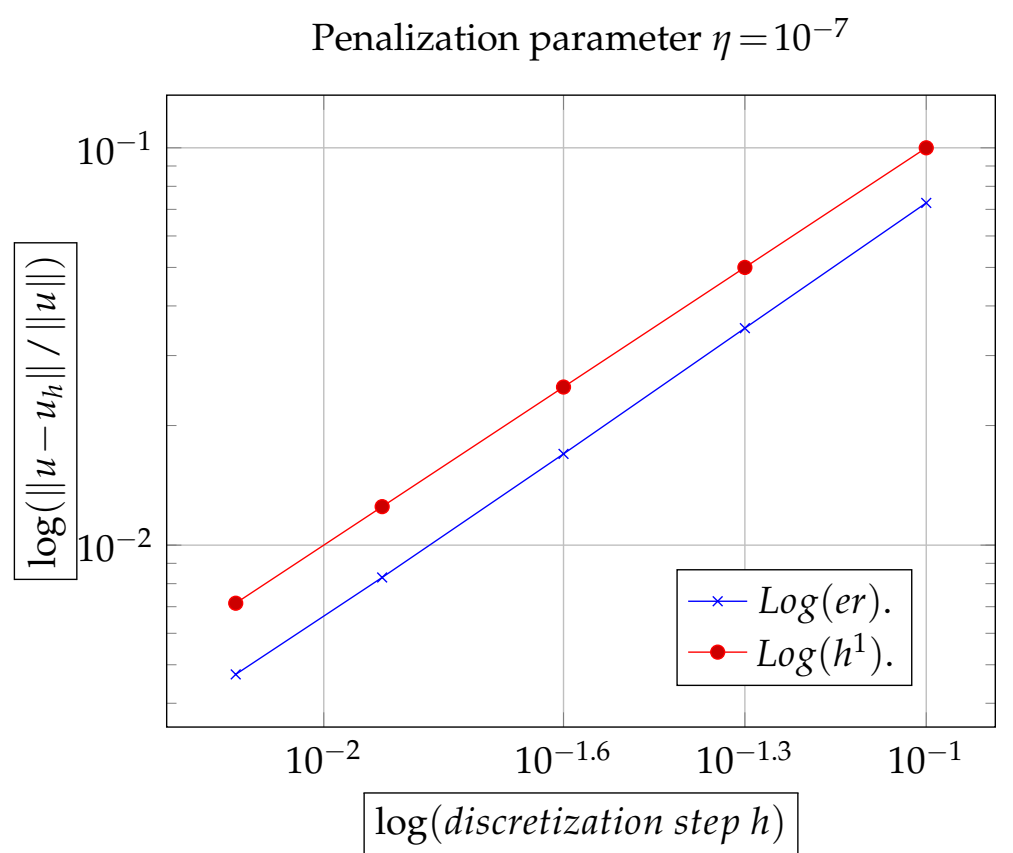

Figure 4: First order accuracy of the J.E.B.C. method (D1) for $\widetilde{\mathcal{P}}_{D H}$ problem.

Table 3: The relative $\mathbb{L}^{2}$ error norms according to the number of discretization points for non homogeneous Dirichlet problem in L-shaped domain.

\begin{tabular}{||c|c|c|c|c|c||}
\hline Mesh step $h$ & $1 / 16$ & $1 / 32$ & $1 / 64$ & $1 / 128$ & $1 / 140$ \\
\hline$\frac{\left\|u_{e x}-u_{h}\right\|}{\left\|u_{e x}\right\|}$ & $1.075866 e-2$ & $4.604882 e-3$ & $2.164175 e-3$ & $1.032435 e-3$ & $9.361342 e-4$ \\
\hline
\end{tabular}

which has the analytic solution $\tilde{u}(x, y)=\tilde{v}(x, y)=x(x-1) \sin (\pi y)$ in $\widetilde{\Omega}$, the table below gives the relative error norm for non homogeneous Dirichlet problem in the L-Shaped Domain (see Table 3).

The fictitious problem over $\Omega$ is solved using J.E.B.C. method (D1). As Fig. 5 shows, the first order a accuracy is reached for $\mathbb{L}^{2}$-norm for exterior approximate interface.

Robin problem. We now consider the Robin problem with an appropriate data of $f$ such that :

$$
\widetilde{\mathcal{P}}_{N} \begin{cases}-\nabla \cdot \sigma(\tilde{u})=\tilde{f} & \text { in } \widetilde{\Omega}, \\ \tilde{u}=0 & \text { on } \widetilde{\Gamma}, \\ \sigma(\tilde{u}) \cdot n=\tilde{u}+\mathbf{g} & \text { on } \Sigma(\mathbf{A}=\alpha I=I, \mathbf{q}=\mathbf{g}),\end{cases}
$$




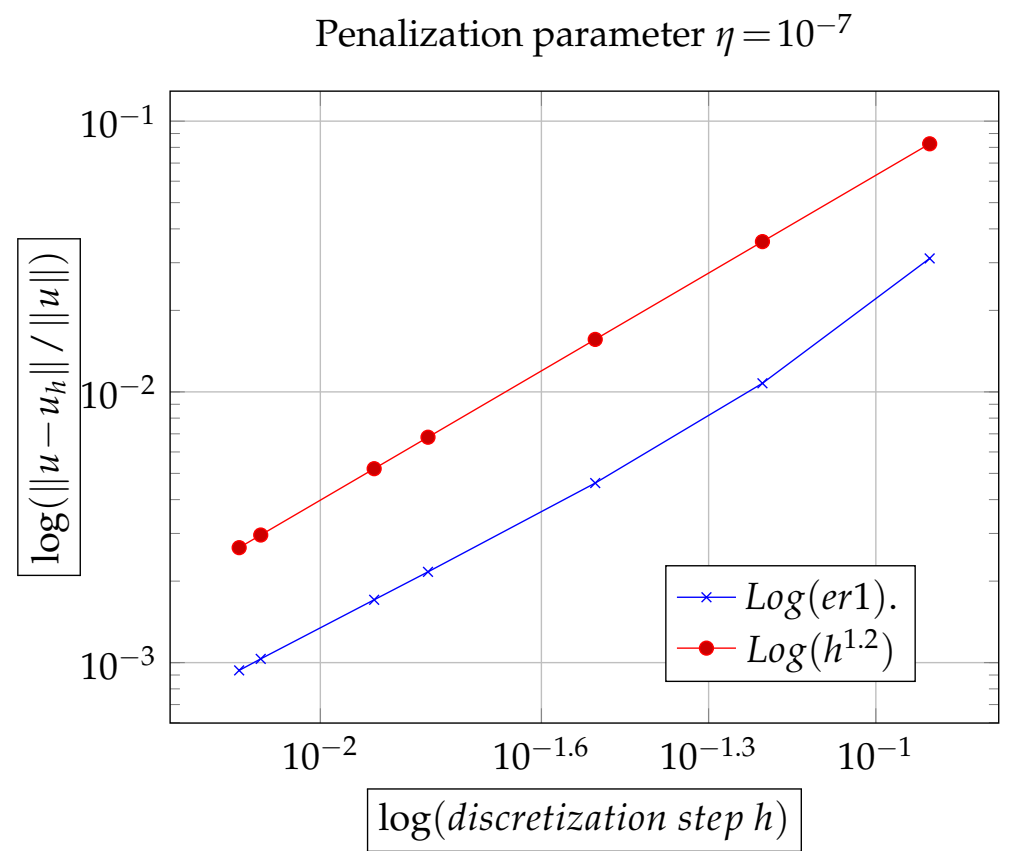

Figure 5: Order of the convergence of the J.E.B.C. method (D1) for $\widetilde{\mathcal{P}}_{D N H}$ problem.

Table 4: Variation of relative $\mathbb{L}^{2}$ error norms according to the number of discretization points for Fourier condition in L-shaped domain.

\begin{tabular}{||c|c|c|c|c|c||}
\hline Mesh step $h$ & $1 / 16$ & $1 / 32$ & $1 / 64$ & $1 / 128$ & $1 / 140$ \\
\hline$\frac{\left\|u_{e x}-u_{h}\right\|}{\left\|u_{e x}\right\|}$ & $4.914116 e-3$ & $1.181075 e-3$ & $2.825945 e-4$ & $6.513587 e-5$ & $5.352028 e-5$ \\
\hline
\end{tabular}

where

$$
\mathbf{g}=\left\{\begin{array}{l}
\left(-\frac{1}{2}(\lambda+2 \mu)+\frac{3 \alpha}{16}\right) \sin (\pi y)+\frac{3}{16} \lambda \pi \cos (\pi y) \\
\left(-\frac{1}{2} \mu+\frac{3 \alpha}{16}\right) \sin (\pi y)+\frac{3}{16} \pi \mu \cos (\pi y)
\end{array}\right.
$$

on the $\Sigma_{x}=\{x=1,3 / 4 \leq y \leq 1\}$ and

$$
\mathbf{g}=\left\{\begin{array}{l}
(-\mu(2 x-1)+\alpha x(x-1)) \sin (3 \pi / 4)-\mu \pi x(x-1) \cos (3 \pi / 4), \\
(-\lambda(2 x-1)+\alpha x(x-1)) \sin (3 \pi / 4)-\pi(\lambda+2 \mu) x(x-1) \cos (3 \pi / 4),
\end{array}\right.
$$

on the $\Sigma_{y}=\{3 / 4 \leq x \leq 1, y=1\}, \Sigma_{x} \cup \Sigma_{y}=\Sigma$. The results obtained are illustrated in the Table 4 . tion.

Fig. 6 shows a second-order accuracy of the J.E.B.C. method (F), for the Robin condi- 


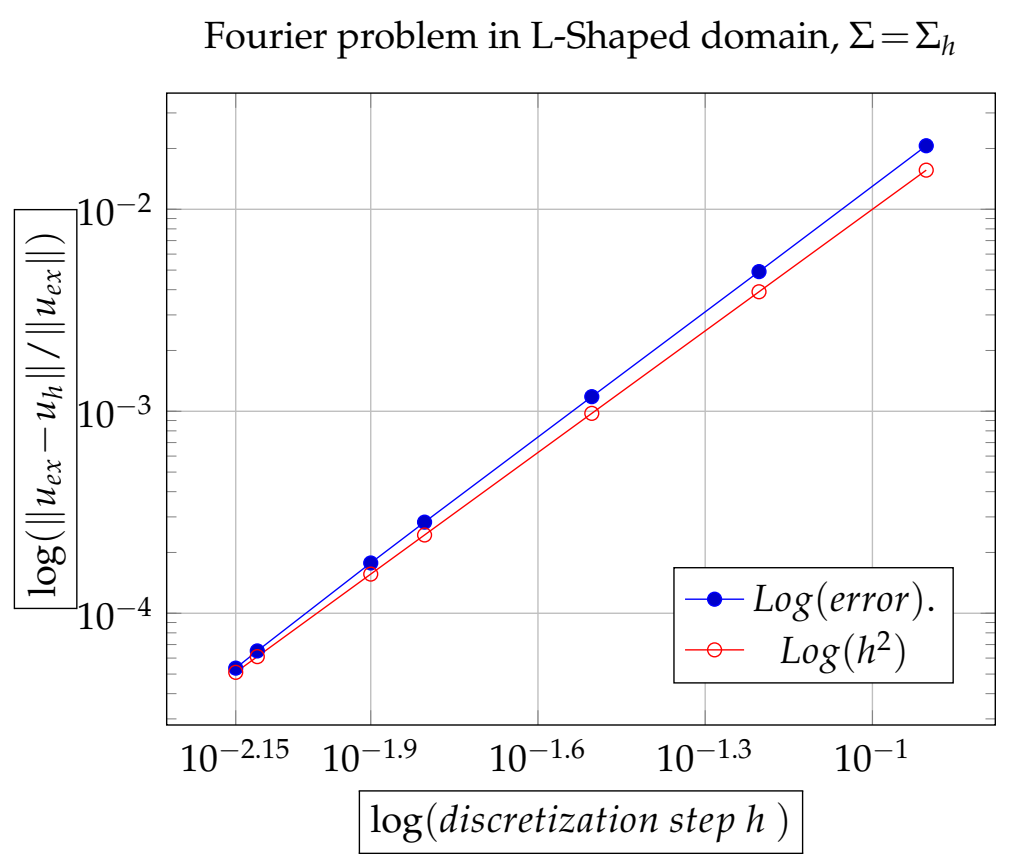

Figure 6: Order of the convergence of the J.E.B.C. method (F) for $\widetilde{\mathcal{P}}_{N}$ problem.

The approximate solution obtained with the Fourier approach and the analytic one at the diagonal square section is represented in Fig. 7. It can be seen that the behavior of the approximate solution at the corner $\left(x=\frac{3}{4}, y=\frac{3}{4}\right)$ of the physical domain $\widetilde{\Omega}$.

\subsubsection{Second test problem. a quarter disk domain}

We now consider a quarter disk domain $\widetilde{\Omega}$ immersed in the unit square $\Omega=[0,1] \times[0,1]$. That defines an immersed interface $\Sigma$ for quarter disk, see Fig. 8 . As in the L-shaped case, the fictitious square domain is meshed with uniform grid step $h$. The approximate interface $\Sigma_{h}$, lying on sides of the mesh, is chosen such that it crosses the physical immersed interface $\Sigma$.

Fig. 8 describes of the quarter disk domain, associated fictitious domain mesh and approximate immersed interface.

Homogeneous Dirichlet case. We consider the below homogeneous Dirichlet Problem with the adapted right-hand side $\tilde{f}$

$$
\widetilde{P}_{D H} \begin{cases}-\nabla \cdot \sigma(\tilde{u})=\tilde{f} & \text { in } \widetilde{\Omega}, \\ \tilde{u}=0 & \text { on } \widetilde{\Gamma}, \\ \tilde{u}=u_{D}=0 & \text { on } \Sigma,\end{cases}
$$




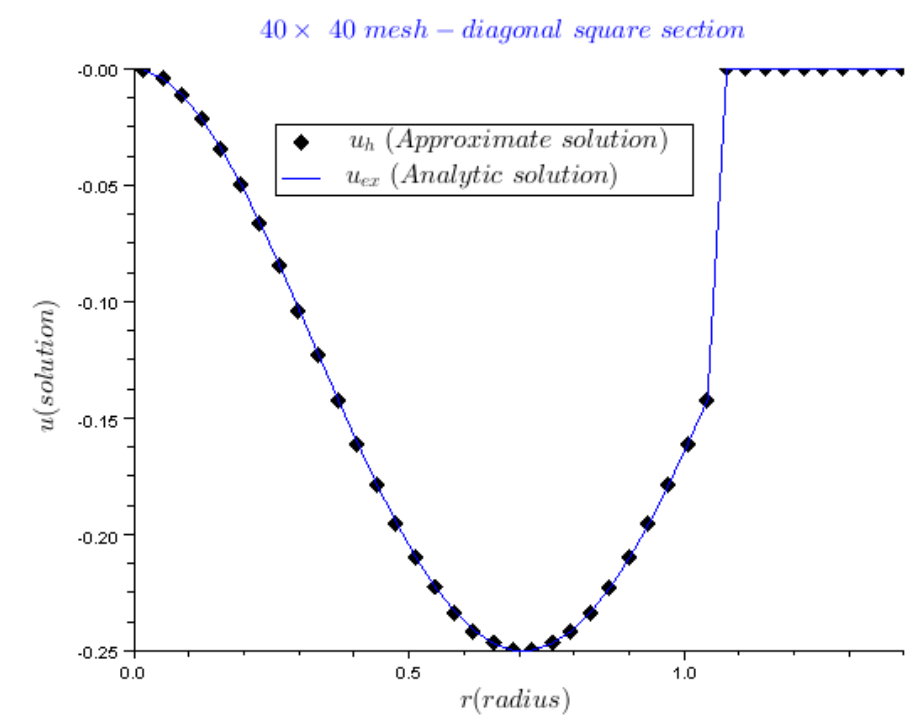

Figure 7: Approximate solution and analytic one in the Fourier case at a diagonal square section.

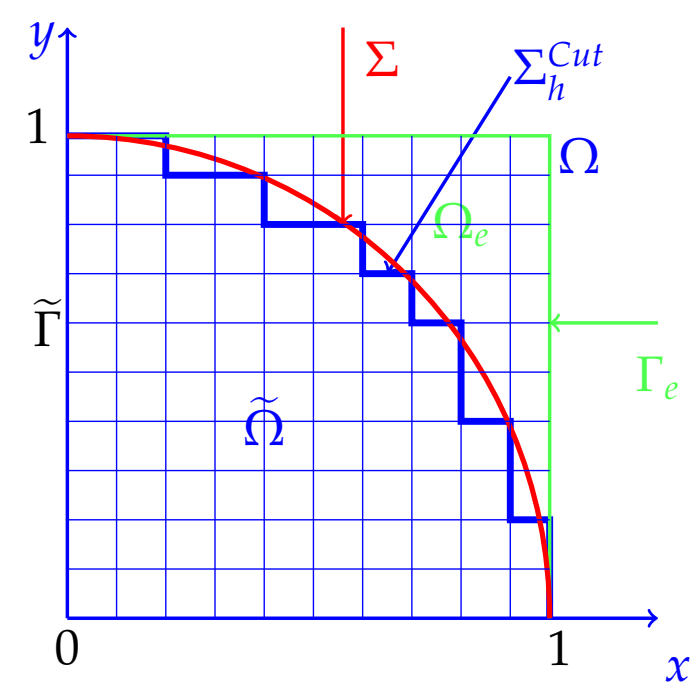

(a)

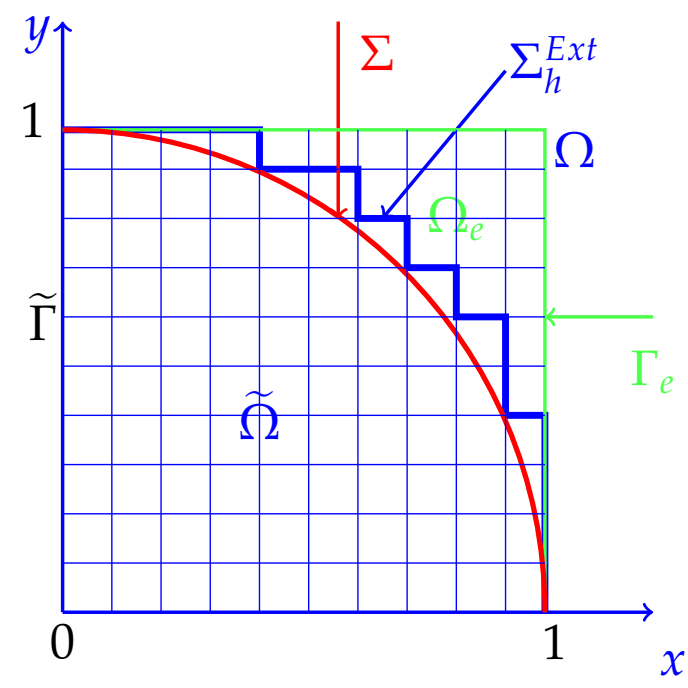

(b)

Figure 8: Approximate interface $\Sigma_{h}$ and approximated domain $\widetilde{\Omega}_{h}$. (a) Cut approximate interface $\Sigma_{h}^{C u t}$, (b) Exterior approximate interface $\Sigma_{h}^{E x t}$.

which has the below analytic solution

$$
\tilde{u}(x, y)=\tilde{v}(x, y)=4 x y\left(1-\exp \left(\frac{1}{4}\left(r^{2}-1\right)\right)\right) \text { in } \widetilde{\Omega}, \quad \text { which } r=\sqrt{x^{2}+y^{2}},
$$


Table 5: Variation of relative $\mathbb{L}^{2}$ error norms according to parameter $h$ for homogeneous Dirichlet problem in the quarter disk domain using (D1).

\begin{tabular}{||c|c|c|c|c|c||}
\hline Mesh step $h$ & $\frac{1}{10}$ & $\frac{1}{40}$ & $\frac{1}{80}$ & $\frac{1}{120}$ & $\frac{1}{140}$ \\
\hline$\frac{\left|u_{e x}-u_{h}\right|}{\left|u_{e x}\right|}$ & $6.504512 e-2$ & $2.863497 e-2$ & $9.679815 e-3$ & $3.856029 e-3$ & $3.238966 e-3$ \\
\hline
\end{tabular}

Table 6: Variation of relative $\mathbb{L}^{2}$ error norms according to parameter $h$ for homogeneous Dirichlet problem in the quarter disk domain using (D2).

\begin{tabular}{||c|c|c|c|c|c||}
\hline Mesh step $h$ & $\frac{1}{10}$ & $\frac{1}{40}$ & $\frac{1}{60}$ & $\frac{1}{80}$ & $\frac{1}{120}$ \\
\hline$\frac{\left|u_{e x}-u_{h}\right|}{\left|u_{e x}\right|}$ & $6.494105 e-2$ & $2.470404 e-2$ & $1.024406 e-2$ & $8.240813 e-3$ & $5.464180 e-3$ \\
\hline
\end{tabular}

where $(r, \theta)$ are the polar coordinates at the origin. Table 5 gives the relative error norms for homogeneous Dirichlet problem in square domain, with $\eta=\frac{h}{2(\lambda+2 \mu)}$, using J.E.B.C. method (D1) with the cut approximate interface $\Sigma_{h}^{c u t}$.

Now, we use the following variant $\mathbf{g}=\mathbf{q}=0$ and $\mathbf{M}=\mathbf{S}=\frac{1}{\eta}$, (see D2 in Table 1).

The following computations are performed with $\eta=1 e-10$ to get the modeling error negligible compared to the discretization error using J.E.B.C. method (D2) with cut approximate interface $\Sigma^{\text {cut }}$ (see Table 6).

Fig. 9. Convergence for the $\mathbb{L}^{2}$-norm of the discretization error for the homogeneous

The J.E.B.C. methods with cut interface $\Sigma_{h}^{c u t}$

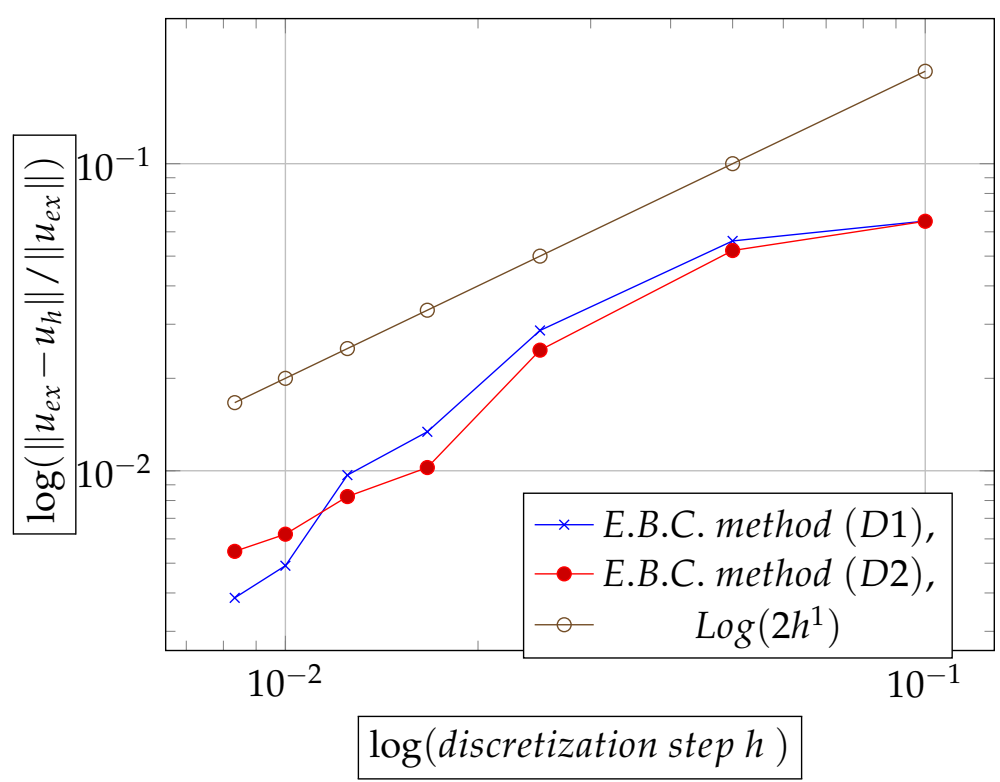

Figure 9: The first order accuracy for (D1) and (D2) methods with cut interface in a quarter disk domain. 
Table 7: Variation of relative $\mathbb{L}^{2}$ error norms according to parameter $h$ for non homogeneous Dirichlet problem in the quarter disk domain using (D1).

\begin{tabular}{||c|c|c|c|c|c||}
\hline Mesh step $h$ & $\frac{1}{20}$ & $\frac{1}{40}$ & $\frac{1}{60}$ & $\frac{1}{80}$ & $\frac{1}{120}$ \\
\hline$\frac{\left|u_{e x}-u_{h}\right|}{\left|u_{e x}\right|}$ & $3.026736 e-1$ & $1.168293 e-1$ & $9.872092 e-2$ & $6.252881 e-2$ & $3.941031 e-2$ \\
\hline
\end{tabular}

Dirichlet elasticity problem with $\Sigma_{h}^{c u t}$ and using (D1) and D2 J.E.B.C. methods.

Non homogeneous Dirichlet case. We consider the below non homogeneous Dirichlet Problem defined in $\widetilde{\Omega}$

$$
\widetilde{P}_{D N H} \begin{cases}-\nabla \cdot \sigma(\tilde{u})=\tilde{f} & \text { in } \widetilde{\Omega}, \\ \tilde{u}=0 & \text { on } \widetilde{\Gamma}, \\ \tilde{u}=u_{D}=\frac{3}{2} \sin (2 \theta) & \text { on } \Sigma,\end{cases}
$$

where $\theta=\arctan \left(\frac{y}{x}\right)$, has the analytic solution

$$
\tilde{u}(x, y)=\tilde{v}(x, y)=x y\left(4-r^{2}\right) \text { in } \widetilde{\Omega} \text {, which } r=\sqrt{x^{2}+y^{2}} .
$$

The computations are illustrated in Table 7. In Fig. 10 we show a log-log plot of the errors both displacement components $u$ and $v$ versus the discretization step $h$. Also shown is a straight with slope of 1 which corresponds to first order accuracy for the method (D1).

The approximate solution obtained with the J.E.B.C. method (D1) and the analytic one at the diagonal square section is represented in Fig. 11.

Robin or non homogeneous Neumann case. When the interface $\Sigma$ is approximated by the mesh volume edges into $\Sigma_{h}$, the non-homogeneous Neumann (or Robin) embedded boundary condition case requires the surface correction in the scalar case, as shows the test below. But in the vectorial one, the surface correction is not required

$$
\widetilde{\mathcal{P}}_{N} \begin{cases}-\nabla \cdot \sigma(\tilde{u})=\tilde{f} & \text { in } \widetilde{\Omega}, \\ \tilde{u}=0 & \text { on } \widetilde{\Gamma}, \\ \sigma(\tilde{u}) \cdot n=\mathbf{q} & \text { on } \Sigma .\end{cases}
$$

We have chosen $\tilde{f}$ such that the problem $\left(\widetilde{\mathcal{P}}_{N}\right)$ has the analytical solution

$$
\tilde{u}(x, y)=\tilde{v}(x, y)=4 x y\left(1-\exp \left(\frac{1}{4}\left(x^{2}+x^{2}-1\right)\right)\right) \quad \text { in } \widetilde{\Omega},
$$

where

$$
q=\left\{\begin{array}{l}
-2\left((\lambda+2 \mu) y\left(1-y^{2}\right)+\lambda y^{2} \sqrt{1-y^{2}}\right) \mathbf{n}_{x}-2 \mu\left(x\left(1-x^{2}\right)+x^{2} \sqrt{1-x^{2}}\right) \mathbf{n}_{y} \\
-2 \mu\left(y\left(1-y^{2}\right)+y^{2} \sqrt{1-y^{2}}\right) \mathbf{n}_{x}-2\left((\lambda+2 \mu) x\left(1-x^{2}\right)+\lambda x^{2} \sqrt{1-x^{2}}\right) \mathbf{n}_{y} .
\end{array}\right.
$$




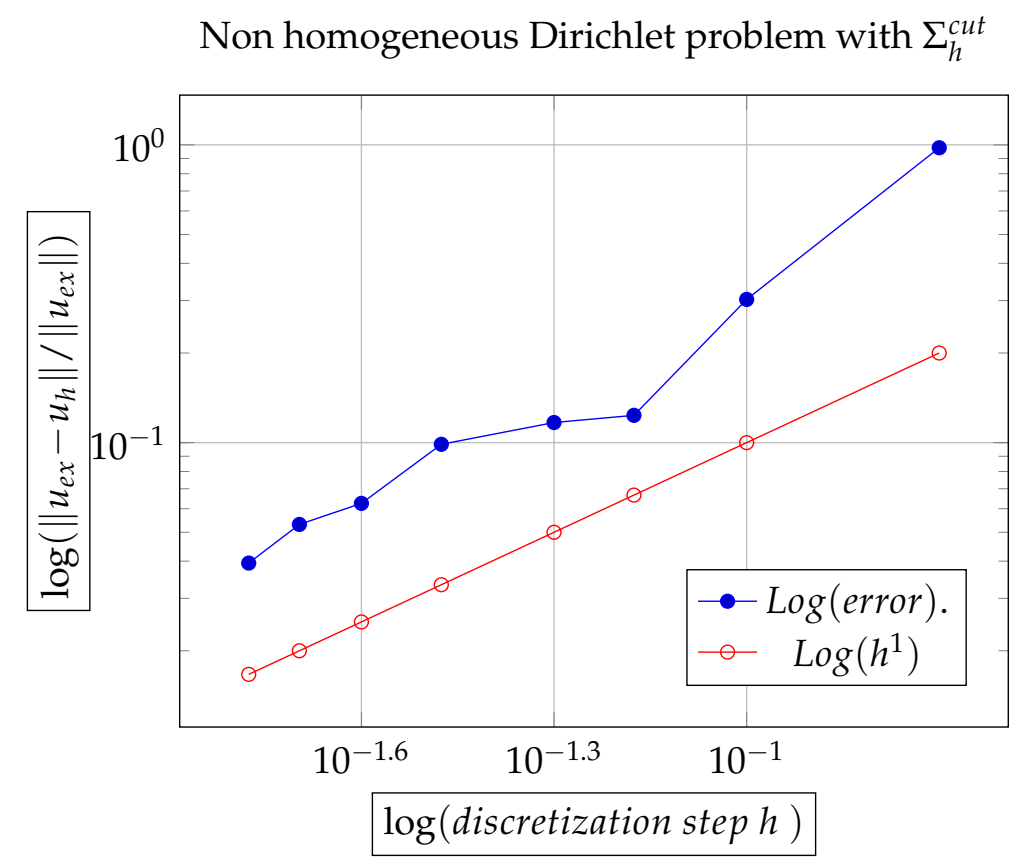

Figure 10: The first order accuracy for (D1) J.E.B.C. method for non homogeneous Dirichlet problem.

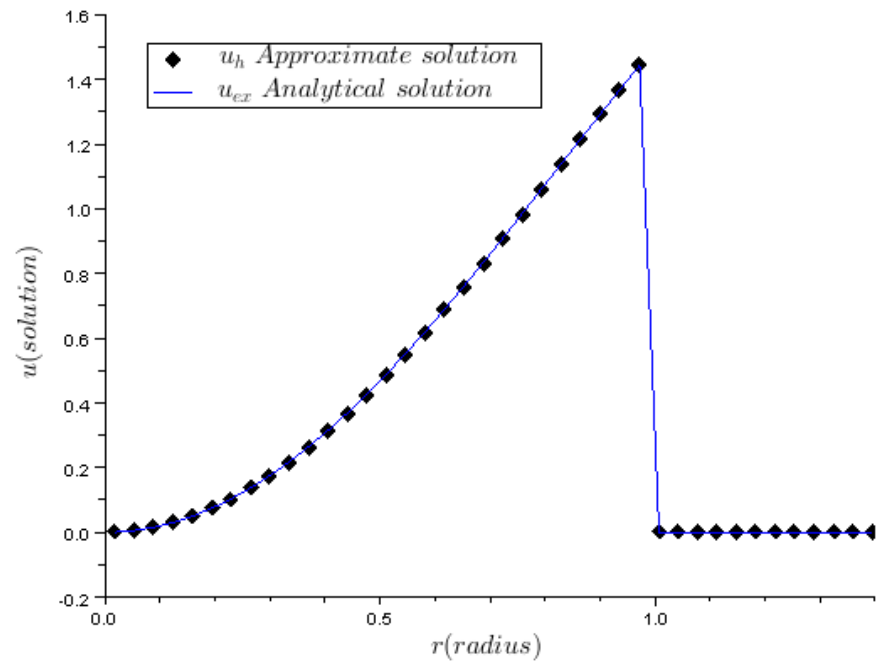

Figure 11: Approximate solution and analytical one in the non homogeneous Dirichlet case at a diagonal square section.

The fictitious domain is solved in $\Omega$ with the J.E.B.C. method $(F)$ without exterior control, as described in the Table 1 , the results for the relative $\mathbb{L}^{2}$-norm error are registered in the 
The J.E.B.C. method (F) of Neumann problem with Cut interface $\Sigma_{h}^{c u t}$

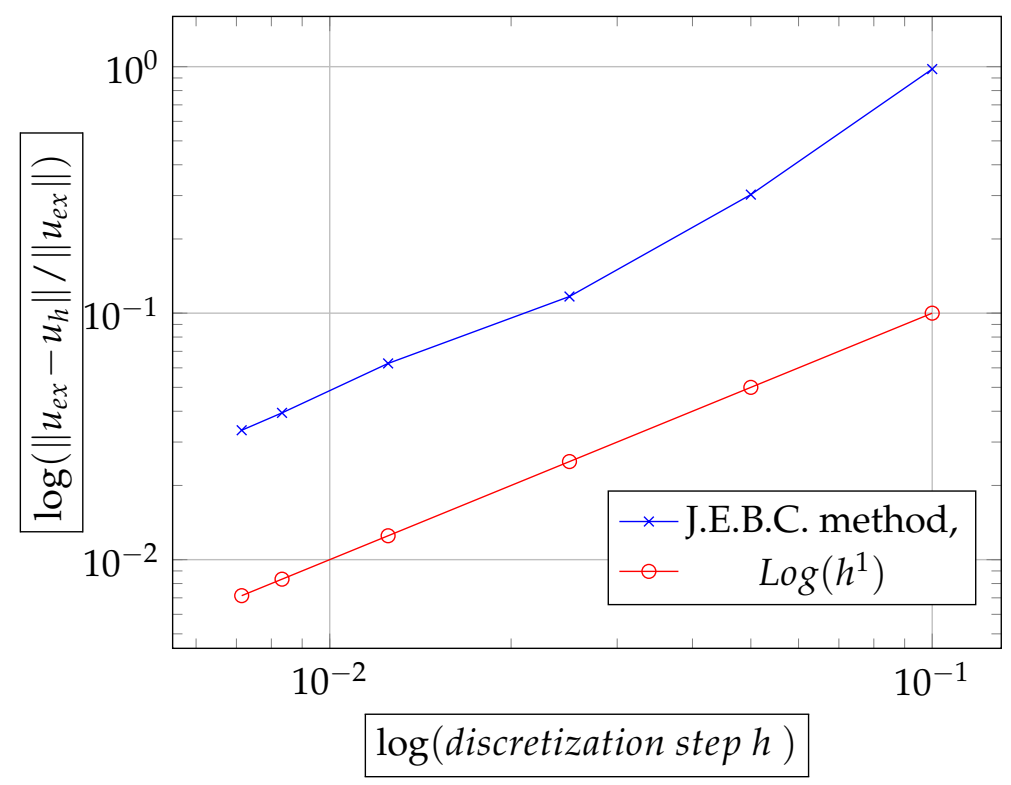

Figure 12: The first order accuracy of the J.E.B.C. Method (F).

Table 8: Variation of relative $\mathbb{L}^{2}$ error norms according to parameter $h$ for a Fourier problem in the quarter disk domain using $(F)$.

\begin{tabular}{||c|c|c|c|c|c||}
\hline Mesh step $h$ & $\frac{1}{10}$ & $\frac{1}{20}$ & $\frac{1}{40}$ & $\frac{1}{80}$ & $\frac{1}{140}$ \\
\hline$\frac{\left\|u_{e x}-u_{h}\right\|}{\left\|u_{e x}\right\|}$ & $9.777809 e-1$ & $3.026692 e-1$ & $1.168142 e-1$ & $6.250331 e-2$ & $3.349663 e-2$ \\
\hline
\end{tabular}

Table 8.

Finally, In order to validate the J.E.B.C. method (F) for any Robin boundary conditions, we now study a Fourier problem

$$
\widetilde{\mathcal{P}}_{F} \begin{cases}-\nabla \cdot \sigma(\tilde{u})=\tilde{f} & \text { in } \widetilde{\Omega}, \\ \tilde{u}=0 & \text { on } \widetilde{\Gamma} \\ \sigma(\tilde{u}) \cdot n=u+\mathbf{q} & \text { on } \Sigma,\end{cases}
$$

which has the analytic solution

$$
\tilde{u}(x, y)=\tilde{v}(x, y)=x y\left(4-\left(x^{2}+y^{2}\right)\right) \text { in } \widetilde{\Omega} .
$$

The fictitious domain problem is solved in $\Omega$ with the J.E.B.C. method $(F)$ without exterior control, as described in Table 1. We investigate in Fig. 13 the effect of the cut approximate interface $\Sigma_{h}^{c u t}$ and exterior one $\Sigma_{h}^{e x t}$. We observe the first-order accuracy for the $\mathbb{L}^{2}$-norm for both approximated immersed interfaces $\Sigma_{h}^{c u t}$ and $\Sigma_{h}^{\text {ext }}$, since the method involving the cut interface is more accurate than the exterior one (see Fig. 13). 
The J.E.B.C. method (F) of Fourier problem

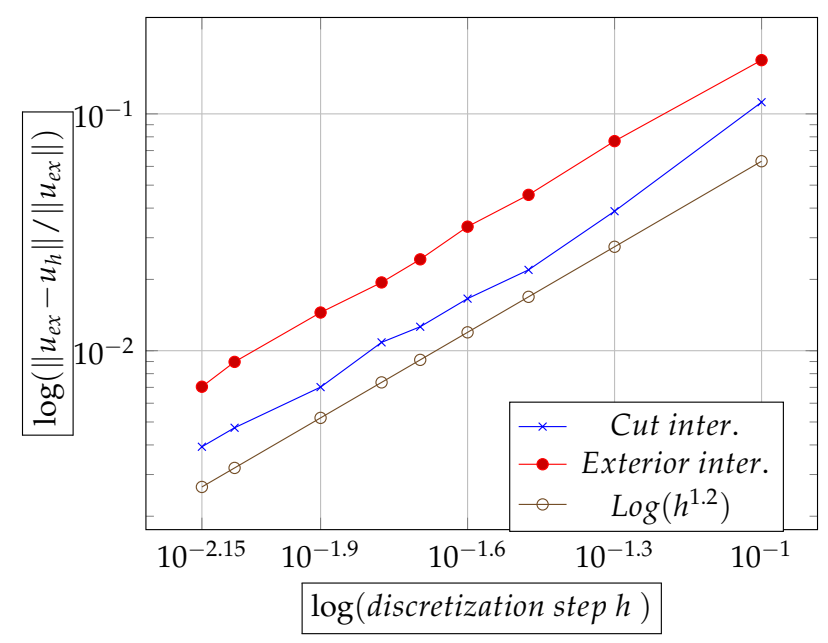

Figure 13: Convergence for the $\mathbb{L}^{2}$-norm of the discretization error with $h$ of the J.E.B.C. method $(F)$ for the Fourier problem with $\Sigma_{h}^{c u t}$ or $\Sigma_{h}^{\text {ext }}$.

\section{Conclusions and perspectives}

An approach of the fictitious domain method to solve linear elasticity problems has been introduced which is based on a sharp interface approach and treats boundary conditions General immersed (Dirichlet, Neumann and Robin). A numerical finite volume scheme with jumps of flux and solution through the immersed interface is used to calculate the solution. The main advantage of this method is its low cost. Indeed, the numerical solution is calculated on a simple Cartesian grid without locally modifying the numerical scheme or introducing an unknown local. This method is a first-order accuracy.

In the next step of this work, we shall introduce another fictitious domain method based on a diffuse interface approach and we will use a numerical finite element scheme to deal with general immersed boundary conditions. These two fictitious domain methods remain of the first order. Then, each method will be combined with a local multi-level algorithm refinement mesh to increase the accuracy of the solution.

The other perspective will be to extend these methods in the fictitious domain elasticity problems with moving boundary conditions. These fictitious domain methods allow to simulate the moving boundaries with a low computational cost and require no remeshing of the domain.

\section{References}

[1] PH. Angot, A unified fictitious domain model for general embedded boundary conditions, C. R. 
Acad. Sci. Paris, Ser. I Math., 341(11) (2005), pp. 683-688.

[2] PH. ANGOT, A model of fracture for elliptic problems with flux and solution jumps, C. R. Acad. Sci. Paris, Ser. I Math. 337(6) (2003), pp. 425-430.

[3] PH. Angot, On the well-posed coupling between free fluid and porous viscous flows, Appl. Math. Lett., 24 (2011), pp. 803-810.

[4] PH. ANGOT, A fictitious domain model for the Stokes/Brinkman problem with jump embedded boundary conditions, C. R. Acad. Sci. Paris, Ser. I, 348 (2010), pp. 697-702.

[5] I. BIjelonja, I. Demirdzic, AND S. MuZAFERIJA, A finite volume method for incompressible linear elasticity, Comput. Methods Appl. Mech. Eng., 195 (2006), pp. 6378-6390.

[6] E. BuRMAN, AND P. HANSBO, Fictitious domain finite element methods using cut elements: I. A stabilized Lagrange multiplier method, Comput. Methods Appl. Mech. Eng., 199 (2010), pp. 2680-2686.

[7] S. Delcourte, Développement de Méthodes de Volumes Finis Pour la Mécanique des Fluides, Thèse de Doctorat de l'université de Toulous, 2007.

[8] I. DemiRDZIC, AND D. MARTINOviC, Finite volume method for thermo-elasto-plastic, Comput. Methods Appl. Mech. Eng., 109 (1993), pp. 331-349.

[9] V. GIRAULT, AND R. GLOWINSKI, Error analysis of a fictitious domain method applied to a Dirichlet problem, Japan J. Indust. Appl. Math., 12(3) (1995), pp. 487-514.

[10] A. HANSBO, AND P. HANSBO, A finite element method for the simulation of strong and weak discontinuities in solid mechanics, Comput. Methods Appl. Mech. Eng., 193 (2004), pp. 35233540.

[11] P. Hansbo, AND M. G. LARson, Discontinuous Galerkin and the Crouzeix-Raviart element: Application to elasticity, M2AN, 37 (2003), pp. 63-72.

[12] H. JASAK, AND H. G. WELleR, Application of the Finite Volume Method and Unstructured Meshes to Linear Elasticity, Elsevier, (1998).

[13] K. Khadra, Ph. Angot, S. Parneix, And J.-P. Caltagirone, Fictitious domain approach for numerical modilling of Navier-Stokes equations, Int. J. Numer. Math. Fluids, 34(8) (2000), pp. 651-684.

[14] J.-L. LiOnS, Problèmes aux Limites dans les équations aux Dérivées Partielles, Presses de l'Université de Montreal, 1965.

[15] S. MesBAHI, AND N. AlAA, Mathematical analysis of a reaction diffusion model for image restoration, Ann. Univ. of Craiova, Math. Comput. Sci. Ser., 42(1) (2015), pp. 70-79.

[16] I. RAMIÈRE, PH. ANGOT, AND M. BELLIARD, A fictitious domain approach with spread interface for elliptic problems with general boundary condition, Comput. Methods Appl. Mech. Eng., 196 (2007), pp. 766-781.

[17] I. Ramière, PH. ANGot, AND M. Belliard, A general fictitious domain method with immersed jumps and multilevel structured meshes, J. Comput. Phys., 225 (2007), pp. 1347-1387.

[18] Y. SAAD, Iterative Methods for Sparse Linear Systems, Second Edition, SIAM Publications, 2003.

[19] A. Sarthou, S. Vincent, Ph. Angot, And J. P. Caltagirone, The sub-mesh penalty method, Finite Volumes for Complex Applications V, R. Eymard and J.-M. Hérard (Eds), pp 633-640, ISTE Ltd and J. Wiley \& Sons, 2008.

[20] T. Ye, R. MitTAL, H. S. UdAYKUMAR, AND W. ShyY, An accurate Cartesian grid method for viscous incompressible flow with complex immersed boundaries, J. Comput. Phys., 156 (1999), pp. 209-240. 